\title{
Perfil metabólico de ovelhas em gestação
}

\author{
Brondani, W.C. ${ }^{1} ;$ Lemes, J.S. ${ }^{\text {; }}$ Ferreira, O.G.L. ${ }^{2}$; Roll, V.F.B. ${ }^{2}$ e Del Pino, F.A.B. ${ }^{3}$
}

'Programa de Pós-Graduação em Zootecnia (PPGZ)/Universidade Federal de Pelotas. UFPel. Brasil. ${ }^{2}$ Departamento de Zootecnia e Ciências Biológicas. UFSM. Campus Palmeira das Missões. Brasil. ${ }^{3}$ Departamento de Bioquímica. Universidade Federal de Pelotas. UFPel. Brasil.

\section{PALAVRAS ChAVE ADICIONAIS}

\section{Ovinocultura.}

Energia.

Proteína.

Minerais.

\section{RESUMO}

A identificação do perfil metabólico em animais de produção atua como um método auxiliar na avaliação de rebanhos com diferentes índices produtivos e reprodutivos, assim como uma importante ferramenta no diagnóstico clínico de doenças do metabolismo que podem afetar o desempenho produtivo dos animais. O objetivo deste estudo é realizar um levantamento de informações sobre o perfil metabólico energético, proteico e mineral de ovelhas em gestação, avaliando o que já foi estudado sobre o tema e salientando o que ainda necessita mais pesquisas. Dentre os metabólitos utilizados para avaliação do status nutricional dos ruminantes, estão a glicose, o beta-hidroxibutirato (BHB), os ácidos graxos livres (NEFA) e o colesterol para avaliação energética, a ureia, a albumina e as proteínas totais para avaliação protéica, e para avaliação mineral cálcio, fósforo e magnésio. Esta revisão disserta a influência do perfil metabólico no desempenho produtivo de ovelhas gestantes, valores de referência e estudos relacionados. No entanto existem poucos estudos sobre o efeito das alterações metabólicas gestacionais sobre a estrutura da fibra muscular, desenvolvimento, crescimento e qualidade da carne dos cordeiros gerados. Assim, há necessidade de novas pesquisas voltadas a esclarecer questões relacionadas com esse tema.

\section{Metabolic profile of sheep in pregnancy}

\section{SUMMARY}

The identification of the metabolic profile in animal production acts as a method for the evaluation of herds with different production and reproductive index as well as an important tool in clinical diagnosis of metabolic diseases that can affect the productive performance of animals. The aim of this study was to conduct a survey of information on energy, protein and mineral metabolic profile of pregnant sheep, assess what has been studied about the topic and highlighting what still needs to be further investigated. Among the metabolites used to assess the nutritional status in ruminants, glucose, beta- hydroxybutyrate (BHB), free fatty acids (FFA) and cholesterol are evaluated to measure the energy state; urea, albumin and total protein are used for evaluation of protein and calcium, phosphorus and magnesium to measure mineral condition. This review covers the influence of the metabolic profile in the growth performance of pregnant ewes, and related studies. However there are few studies on the effect of gestational metabolic alterations on the structure of muscle fiber development, growth and meat quality of lambs. Therefore, more research is required in order to clarify the issues related to this topic.

\section{INFORMACIÓN}

Cronología del artículo.

Recibido/Received: 23.05 .2014

Aceptado/Accepted: 16.11.2015

On-line: 17.02 .2016

Correspondencia a los autores/Contact e-mail:

wcbrondani@hotmail.com

\section{INTRODUÇÃO}

A alimentação é um fator que influencia o crescimento animal. Assim, dependendo de sua severidade, duração e momento em que ocorre na ovelha gestante, a restrição alimentar pode afetar o crescimento fetal e o peso do cordeiro ao nascimento. Os períodos mais críticos para sua ocorrência são a fecundação e a fase final da gestação, quando a placenta diminui sua capa- cidade de compensar o fornecimento inadequado de nutrientes (Cañeque et al., 1989). A gestação em ovinos é um período critico devido ao aumento das necessidades nutricionais e consequentemente incremento da mobilização de nutrientes para o desenvolvimento do úbere e manutenção do organismo (El-Sherif and Assad, 2001). Durante as últimas seis semanas antes do parto, os requerimentos nutricionais das matrizes aumentam, momento em que se acelera o desenvol- 
vimento fetal e completam-se aproximadamente $70 \%$ do seu crescimento (Russel, 1979). Nesta fase, é importante iniciar estratégias de manejo que garantam as necessidades nutricionais das ovelhas (Mexia et al., 2006), tendo em vista que a nutrição inadequada durante a gestação poderá limitar a capacidade de crescimento pós-natal dos músculos esqueléticos dos cordeiros (Greenwood et al., 2000; Patiño and Van Cleef, 2010).

Um importante marcador da condição nutricional é o escore de condição corporal (ECC), que também pode estar associado às condições de saúde dos rebanhos (Roche et al., 2009). Smith and Sherman (2009) sugerem que ovelhas devem estar com condição corporal de 3,0 a 3,5 (escala de 1-5) no terço final da gestação, 3,5 no parto e de 2,0 a 2,5 no desmame para que não tenham deficit energético. Outra maneira de avaliar a condição nutricional de um rebanho é mediante a determinação de alguns metabólitos sanguíneos. A identificação do perfil metabólico em animais de produção atua como um método auxiliar na avaliação de rebanhos com diferentes índices produtivos e reprodutivos, assim como uma importante ferramenta no diagnóstico clínico de doenças do metabolismo (Peixoto and Osório, 2007). O perfil metabólico vem sendo estudado por diversos autores que relatam redução dos metabólitos com o avanço da gestação ou da lactação em função de um balanço nutricional negativo (Althaus et al., 1995; Contreras and Wittwer, 2000; González 2000; Ribeiro et al. 2003; Cardoso et al., 2011), o que pode afetar diretamente o desempenho produtivos dos animais.

Assim, o objetivo desta revisão é realizar um levantamento de informações sobre o perfil metabólico energético, proteico e mineral de ovelhas em gestação, avaliando o que já foi estudado sobre o tema e salientando o que ainda necessita mais estudos.

\section{ALTERACÕ̃ES DO PERFIL METABÓLICO DURANTE O PERI-PARTO}

Em ovinos, os distúrbios do metabolismo do peri-parto causam perdas econômicas significativas ao produtor, pois podem reduzir a produção leiteira, diminuir o ganho de peso do cordeiro e até mesmo promover a sua morte (Cardoso et al., 2011).

Neste sentido, estudos como os de Roche et al. (2009) indicam que fêmeas com ECC ao parto maior que 3,5 (em uma escala de 1 a 5) possuem altos níveis de ácidos graxos não-esterificados (AGNE) no início da lactação, o que pode levar ao comprometimento das funções hepáticas (Barnett et al., 1988) e imunes (Nikolajczyk et al., 2011), além de diminuição da sensibilidade à insulina pelos tecidos periféricos (Garvey and Birnbaum, 1993; Muligan et al., 2006).

Embora no periparto de ruminantes, três semanas antes e três após o parto, a diminuição da sensibilidade à insulina, também chamada resistência à insulina, ocorra de forma fisiológica (Regnault et al., 2004), esta pode ser agravada em condições de desequilíbrio nutricional, assim como pelo grau de adiposidade ao parto, descrito anteriormente por Oikawa and Oetzel (2006). Tal condição, associada a altas concentrações plasmáticas de ácidos graxos não esterificados (AGNE), tem sido proposta como fator de risco para síndromes metabólicas como a cetose e hipocalcemia em ovinos (Schlumbohm and Harmeyer, 2003; Husted et al., 2008).

Em humanos, diferentes intensidades de resistência à insulina também estão associadas às síndromes metabólicas (Hauner, 1995), sendo uma delas, a presença de cálculos renais devido ao aumento da excreção renal de cálcio e magnésio em pacientes portadores de diabetes tipo II (Jawerbaum and White, 2010). Embora pouco esclarecido, sabe-se que a insulina atua diretamente no túbulo contorcido distal do rim, aumentando a excreção de cálcio e magnésio, levando à retenção de sódio (Na) e potássio (K). Tal mecanismo passa a ser particularmente importante nos pacientes insulino dependentes portadores de hipertensão (Lee et al., 2006).

Por estes motivos a insulina também tem sido proposta como hormônio de regulação do metabolismo do magnésio (Barbagallo et al., 2003), hipótese que é reforçada por estudos in vitro demonstrando acúmulo de $\mathrm{Mg}$ e K em células da musculatura lisa uterina após tratamento com este hormônio (Lostroh and Krathl, 1973). Além disso, em humanos, a hiperglicemia presente em diabéticos está relacionada à hipermagnesúria, levando a queda nas concentrações plasmática de Mg (Mcnair et al., 1978). Por outro lado, a ingestão de altas quantidades de Mg também parece estar relacionada ao aumento da sensibilidade à insulina, o que reforça a relação destes metabólitos (Mooren et al., 2011). Estes achados podem ter importância na ocorrência de hipocalcemia e hipomagnesemia em ruminantes alimentados com alto nível de energia no pré-parto, com consequente efeito negativo direto na produção leiteira e no desempenho reprodutivo pós-parto (Mulligan et al., 2006). Apesar da condição corporal pré-parto ser um fator de risco para ocorrência de hipocalcemia, o mecanismo desta associação não está bem esclarecido. Entretanto, a relação da hipomagnesemia e hipocalcemia está bem caracterizada devido à participação do magnésio como cofator enzimático na ligação e sinalização celular do paratormônio (PTH) e seu receptor (Goff, 2008).

Os efeitos da resistência à insulina, e suas possíveis consequências no metabolismo de cálcio e magnésio durante o pré-parto, também podem afetar outros aspectos produtivos em ruminantes, já que o terço final da gestação corresponde ao maior crescimento do feto e muitas dessas alterações metabólicas podem ter consequências na vida produtiva do animal gerado (Montossi et al., 1998).

Em camundongos foi verificado que os níveis de magnésio, em mães com resistência à insulina, durante o pré e pós-parto influenciaram a adiposidade corporal e à secreção de insulina ao longo da vida dos filhotes (Venu et al., 2008). Estudos realizados em humanos, principalmente em indivíduos com diabetes tipo II, foi observado que a diminuição de insulina em situação de doença, promove diminuição nas fibras musculares tipo I (lenta) e aumento nas fibras tipo II (rápida), além de aumento nas enzimas glicolíticas, sugerindo que ocorre um mecanismo compensatório na captação da glicose pelo músculo em virtude da alteração no metabolismo da glicose (Nyholm et al., 1997; Oberbach et al., 2006; Venojärvi et al., 2005). 


\section{INDICADORES DO PERFIL METABÓLICO DE OVINOS}

Ao estudarem as variações da composição sanguínea de ovelhas no pré e no pós-parto, Del Valle et al. (1983), concluíram que os componentes que melhor expressaram as variações do estado nutricional foram a hemoglobina (HB), o hematócrito (VG) e a glicose, os quais diminuíram quando os requerimentos nutricionais aparentemente não foram preenchidos.

Segundo Peixoto and Osório (2007), a glicose é o metabólito de eleição para avaliar o status energético dos ruminantes, por representar a via metabólica da energia. Porém o déficit energético deve ser muito intenso para que diminua a concentração de glicose sanguínea (González, 2000). Para compensar a falta de glicose, triglicerídeos são mobilizados resultando em aumento nos níveis plasmáticos dos ácidos graxos não esterificados (NEFA). A quebra do NEFA aumenta a concentração do betahidroxibutirato no plasma, inibindo os níveis da gliconeogênese hepática e aumentando a hipoglicemia maternal. Assim, outros metabólitos sanguíneos, como o beta-hidroxibutirato (BHB) e os ácidos graxos livres (NEFA), também são utilizados para determinar o nível nutricional dos ruminantes. Ambos indicadores estão relacionados com a taxa de mobilização das reservas lipídicas em situação de balanço energético negativo (Peixoto and Osório, 2007), como ocorre nos casos de toxemia da prenhez. O aumento exacerbado do NEFA no sangue, observado em casos de toxemia da prenhez, está relacionado com a mobilização de triglicerídeos do tecido adiposo e consequentemente a produção excessiva de corpos cetônicos, que se acentua durante a fase final de gestação, fato atribuído à diminuição do consumo de alimentos (Nasciutti, 2011). Ovelhas acometidas, em geral, apresentam alterações como hipercetonemia, cetonúria, hipoglicemia, níveis elevados dos AGNE e cortisol, além do comprometimento da função hepática e renal (Santos et al., 2011).

Contreras and Wittwer (2000) citam que valores superiores a 0,6 e $0,8 \mathrm{mg} / \mathrm{dL}$, respectivamente, para BHB e NEFA, determinam mobilização de gordura em ovinos. O BHB apresenta leves aumentos nas situações em que há balanço energético negativo moderado, entretanto é bastante útil em circunstância onde a demanda de glicose no organismo é critica, como nos caso de inicio de lactação e final de gestação (Nasciutti, 2011).

Ramin et al. (2007), avaliando a concentração de glicose, ureia, cortisol e BHB em ovelhas gestantes no ultimo mês de prenhez, observaram que, com um monitoramento, os componentes metabólicos podem reduzir os casos de aborto e toxemia da prenhez.

Banchero et al. (2006), trabalhando com ovelhas gestantes da raça Merino, divididas em grupos de sub alimentadas e bem alimentadas, com $70 \%$ e $110 \%$ de energia metabolizável, respectivamente, observaram que as concentrações de glicose aumentaram em ambos os grupos nos últimos 30 dias de gestação, sendo que as ovelhas bem alimentadas obtiveram maiores níveis de glicose e consequentemente menores níveis de BHB do que as sub alimentadas durante o período do tratamento.
Os teores sanguíneos de NEFA são bastante significativos para avaliação do estado energético em ruminantes, respondendo rapidamente às mudanças do consumo do alimento, assim como pode sofrer modificações em função das catecolaminas liberadas durante o estresse.

Ribeiro et al. (2003) encontraram, em borregas Corriedale mantidas em pastagem natural durante o verão, baixo teor plasmático de glicose $(49,29 \mathrm{mg} /$ $\mathrm{dL})$ e alto teor de $\operatorname{BHB}(0,79 \mathrm{mg} / \mathrm{dL})$, o que sugere que esse animais tiveram um balanço energético negativo nessa época, e portanto devem ter mobilizado reservas corporais, concluindo que esse valores podem estar relacionados com a estiagem observada na região naquele ano. Segundo González and Silva (2006) os níveis fisiológicos de glicose em ovinos são entorno de 50-80 g/dL.

Antunovic et al. (2011), trabalhando com ovelhas em diferentes estágios fisiológicos, observaram maiores valores séricos de glicose em ovelhas não gestantes de que gestantes e em lactação, 3,86; 3,51 e 3,26 respectivamente, atribuindo esses resultados ao requerimento de glicose pelo organismo, nos diferentes estágios fisiológicos. Já Firat and Ozpinar (2001), não observaram diferenças na concentração de glicose e BHB em ovelhas aos 100 e 120 dias de gestação e inicio de lactação.

No caso do metabolismo proteico, os dois principais indicadores em ruminantes são os níveis séricos de albumina e ureia. A albumina demonstra o estado de nutrição proteica do animal em longo prazo, enquanto a ureia o demonstra em curto prazo (Payne and Payne, 1987).

A albumina é considerada o indicador mais sensível para determinar o status nutricional proteico; valores persistentemente baixos sugerem inadequado consumo de proteína. É a principal proteína plasmática sintetizada no fígado e representa cerca de 50 a $65 \%$ do total de proteínas séricas, além de contribuir com $80 \%$ da osmolaridade do plasma sanguíneo. Entretanto, para detectar mudanças significativas na concentração de albumina, é necessário um período de pelo menos um mês, devido à baixa velocidade de renovação desta proteína. A concentração de albumina sérica é influenciada pelo status proteico, mas é uma variável relativamente insensível devido ao grande tamanho do seu pool e a sua meia-vida relativamente longa (Peixoto and Osório, 2007). Já valores de proteínas totais abaixo do normal, relacionam-se a dietas nutricionais deficientes, o que diminui as concentrações de albumina (Brito et al., 2006).

A ureia é sintetizada no fígado em quantidades proporcionais à concentração de amônia produzida no rúmen e sua concentração está diretamente relacionada com os níveis proteicos da ração e da relação energia / proteína da dieta. A concentração de ureia no sangue pode sofrer alterações durante o dia, principalmente após a alimentação, havendo elevação de seu teor devido à rápida fermentação seguida da absorção de amônia nesse período (Peixoto and Osório, 2007). Segundo Peixoto and Osório (2007), os níveis de uréia sérica encontrados em ovinos tendem a ser sempre mais altos 
que dos bovinos, com valores, conforme González and Silva (2006), de 24-60 mg/dL. Neste sentido, Ribeiro et al. (2003) encontraram durante o ano níveis séricos médios de ureia de 37,97 mg/dL em borregas da raça Corriedale, enquanto Rabassa et al. (2009) obtiveram, em ovelhas, durante o outono inverno níveis médios de 30,68 mg/dl. Segundo Ribeiro et al. (2004), em ovelhas Border Leicester $\times$ Texel que apresentaram queda no valor da condição corporal do início ao final da gestação (3,30 e 2,11, respectivamente), os teores de albumina sanguínea também diminuíram na metade e final deste período (31,05 e 24,44 g/L, respectivamente). Contudo, os teores de ureia sérica não diferiram significativamente, sendo de $5,87 \mathrm{mmol} / \mathrm{L}$ para as ovelhas na metade da gestação e de $5,59 \mathrm{mmol} / \mathrm{L}$ para as ovelhas em final de gestação. Segundo os autores, isto se deve ao fato de as ovelhas em final de gestação possuirem uma demanda proteica maior para o crescimento fetal e desenvolvimento do úbere, fazendo com que diminuam os teores de albumina, o que não ocorre com os teores de ureia pelo fato desta exprimir diretamente a concentração de amônia no rúmen.

Antunovic et al. (2011), observaram menores concentrações de ureia $(\mathrm{mmol} / \mathrm{L})$, em ovelhas gestantes que em ovelhas lactantes (5,70, e 6,80, respectivamente) e maiores de albumina ( $\mathrm{g} / \mathrm{L}$ ) 30,93 e 28,60, respectivamente, atribuindo esse resultados a maior captação de albumina pelo organismos para a produção de colostro e leite.

Além das biomoléculas orgânicas, o tecido animal também possuem elementos inorgânicos que se encontram em uma proporção de 2 a $5 \%$ do peso total dos animais. Esses elementos, os minerais, têm funções essenciais tanto na estrutura dos tecidos e biomoléculas, como no próprio metabolismo animal (González, 2000). Em relação ao metabolismo mineral, o mesmo é representado pelos teores dos principais macro elementos $\mathrm{Ca}, \mathrm{P}$ e $\mathrm{Mg}$, existindo estreita relação entre o cálcio e fósforo.

Quase todo o cálcio presente no organismo e a maior parte do fósforo estão nos tecidos esqueléticos assim, conforme Pugh (2005), dietas deficientes em cálcio e fósforo podem retardar o crescimento e desenvolvimento dos cordeiros.

O cálcio está intimamente associado ao metabolismo. No plasma, existe em duas formas, livre ionizada e orgânica, a qual está associada a moléculas como proteínas, principalmente albumina, ou ácidos orgânicos. O cálcio total, como é medido no sangue, contém a forma ionizada que é biologicamente ativa, e a forma não ionizada (González, 2000).

O organismo animal apresenta atividade intensa para manter as concentrações de cálcio, principalmente no período de puerpério, devido à produção de colostro e posteriormente de leite, que predispõem ao quadro de hipocalcemia (González and Silva, 2006). Em ovelhas, a hipocalcemia é uma síndrome que ocorre com incidência variável, que está associada a queda do cálcio plasmático. Ocorre mais comumente em ovelhas no final da gestação e início da lactação. A etiologia da doença não está completamente entendida, mas parece começar devido ao requerimento fetal de $\mathrm{Ca}$, adiciona- do com fatores limitantes de suplementação do cálcio (González, 2000; Pugh, 2005; González and Silva, 2006).

A absorção de cálcio no intestino diminui com a idade. Animais mais velhos sofrem redução da capacidade de mobilizar reservas de cálcio quando ocorrem desequilíbrios, sendo, portanto, mais suscetíveis de sofrer hipocalcemia. Outros fatores afetam também a absorção de cálcio, tais como: relação Ca:P nos alimentos, quantidade de proteína na dieta, uma vez que o déficit proteico diminui as absorção de cálcio, ingestão excessiva de magnésio, por competição nas células intestinais, e suplementação de vitamina $\mathrm{D}_{3}$, que aumenta a absorção de cálcio e pode causar calcificação dos tecidos moles (González, 2000).

O fósforo existe em combinações orgânicas dentro das células, mas o principal interesse no perfil metabólico reside no fósforo inorgânico que se apresenta no plasma. Segundo Pugh (2005), a carência de fósforo é a deficiência mineral mais comum em animais criados extensivamente ou em pastagens de inverno. A manutenção do nível de P no sangue é governada pelos mesmos fatores que promovem a assimilação de cálcio. Os níveis de P são variáveis também em função da grande quantidade que se recicla via saliva e sua absorção no rúmem e intestino (González, 2000).

O teor de fósforo em ovinos fica entorno de 4 e $7 \mathrm{mg} / \mathrm{dL}^{-1}$ (Pugh, 2005), situações com teores menores que $4 \mathrm{mg} / \mathrm{dL}$ condizem com deficiência do mineral, que resulta em crescimento lento, baixa fertilidade e apetite depravado.

O controle renal de $\mathrm{Mg}$ esta mais direcionado para prevenir a hipermagnesemia, excretando magnésio pela urina (González, 2000). O magnésio é um micromineral essencial importante na estrutura e no metabolismo do organismo, distúrbios no metabolismo do $\mathrm{Mg}$ podem estar relacionado com insuficiência renal e diabetes (Venu et al., 2008).

A hipomagsenemia, que pode ser considerada uma doença nutricional, geralmente causada pela baixa ingestão de magnesio na dieta. $\mathrm{O} \mathrm{Mg}$ é absorvido no intestino mediante uma sistema de transporte ativo que pode ser interferido pela relação Na:K e ainda pela quantidade de energia, de Ca e de P presentes no alimento (González, 2000). A hipomagsenemia pode causar, além de tetania a hiperexcitabilidade, retenção de placenta, anormalidades da digestão ruminal e diminuição da produção leiteira (Nasciutti, 2011).

De Oliveira et al. (2014), trabalhando com ovelhas em diferentes terços da gestação (inicial, medio e final) encontaram valores de cálcio de 9,39, 9,22 e 8,86, respectivamente, observaram uma diminuição desse mineral no terço final da gestação, o que foi atribuído ao crescimento do feto e à síntese do leite. Já os resultados obtidos para o fósforo foram de 7,15; 7,42 e 5,88, respectivamente, os valores baixos encontrados para esse mineral foi atribuído à deficiência de fósforo no solo e consequentemente na pastagem ingerida pelos animais. 


\section{CONSIDERAÇÕES FINAIS}

A avaliação do perfil metabólico na produção animal é uma importante ferramenta para determinação da condição nutricional dos animais. Em ovelhas gestantes alterações no perfil energético, proteico e mineral poderão ocasionar transtornos no organismo acarretando quedas produtivas e essas refletir sobre a taxa de crescimento dos cordeiros.

No entanto, existem poucos estudos sobre o efeito das alterações metabólicas gestacionais sobre a estrutura da fibra muscular, desenvolvimento, crescimento, e, consequentemente, sobre a qualidade da carne dos cordeiros gerados. Assim, há necessidade de novas pesquisas voltadas a esclarecer questões relacionadas com esse tema.

\section{BIBLIOGRAFIA}

Althaus R.; Roldan V.; Scnefaione, L.; Elizalde E., Sosa, J. y Malinskas, G. 1995. Perfiles metabólicos en ovejas lactantes Corriedale: variación durante la lactancia. Rev Argent Prod Anim, 15: 1055-1058.

Antunovic, Z.; Novoselec, J.; Sauerwein, H.; Speranda, M.; Vegara, M. and Pavic, V. 2011. Blood metabolic profile and some of hormones concentration in ewes during different physiological status. Bulgarian J Agr Sci, 17: 687-695.

Barbagallo, M.; Dominguez, L.J.; Galioto, A.; Ferlisii, A.; Cani, C.; Malfa, L.; Pineo, A.; Busardo. A. and Paolisso, G. 2003. Role of magnesium in insulin action, diabetes and cardio-metabolic syndrome X. Mol Aspects Med, 24: 39-52.

Barnett, C.R.; Flatt, P.R. and Loannides, C. 1988. Role of ketone bodies in the diabetes-induced changes in hepatic mixed-function oxidase activities. Biochim Biophys Acta, 967: 250-254.

Banchero, G.E.; Clariget, R.P.; Bencini, R.; Lindsay, D.R.; Milton, J.T.B. and Martin, G.B. 2006. Endocrine and metabolic factors involved in the effect of nutrition on the production of colostrum in female sheep. Reprod Nutr Dev, 46: 447-460.

Brito, M.A.; González, F.H.D.; Ribeiro, L.A.O.; Campos, R.; Lacerda, L.; Barbosa, P.R. e Bergmann, G. 2006. Composição do sangue e do leite em ovinos leiteiros no sul do Brasil: variações na gestação e lactação. Ciênc Rural, 36: 942-948.

Cañeque, V.; Huidobro, F.R.; Dolz, J.F. y Hernández, J.A. 1989. Producción de carne de cordero. Colección Técnica. Ministerio de Agricultura Pesca y Alimentación. Madrid. 515 pp.

Cardoso, E.C.; De Oliveira, D.R.; Balaro, M.F.A.; Rodrigues, L.F.S. e Brandão, F.Z. 2011. Índices produtivos e perfil metabólico de ovelhas Santa Inês no pós-parto no nordeste do Pará. Rev Bras Cienc Vet, 18: 114-120.

Contreras, P.A. e Wittwer, F. 2000. Uso dos perfis metabólicos no monitoramento nutricional dos ovinos. In: González, F.H.D.; Ospina, H.; Barcelos, J.O. e Ribeiro, L.A.O. (Eds.). Perfil metabólico em ruminantes: Seu uso em nutrição e doenças nutricionais. Gráfica UFRGS. Porto Alegre. 108 pp.

De Oliveira, R.P.M.; Maduro, A.H.P.; Lima, E.S. e De Oliveira, F.F. 2014. Perfil metabólico de ovelhas Santa Inês em diferentes fases de gestação criadas em sistema semi-intensivo no estado do Amazonas. Goiania. Ciênc Anim Bras, 15: 81-86.

Del Valle, J.; Wittwer, F. y Hervé, M. 1983. Estudio de los perfiles metabólicos durante lós periodos de gestación y lactancia en ovinos Romney. Arch Med Vet, 15: 65-72.

El-Sherif, M.M.A. and Assad, F. 2001. Changes in some blood constituints of Bark ewes during pregnancy and lactation under semi arid conditions. Small Ruminant Res, 40: 269-277.

Fırat, A. e Özpınar, A. 2002. Metabolic profile of pre-pregnancy, pregnancy and early lactation in multiple lambing Sakız ewes. Changes in plasma glucose, 3-hydroxybutyrate and cortisol levels. Ann Nutr Metab, 46: 57-61.

Garvey, W.T. and Birnbaum, M.J. 1993. Cellular insulin action and insulin resistance. Baillieres Clin Endocrinol Metab, 7: 785-873.

Goff, J.P. 2008. The monitoring, prevention, and treatment of milk fever and subclinical hypocalcemia in dairy cows. Vet J, 176: 50-57.

González, F.H.D. 2000. Uso do perfil metabólico para determinar o status nutricional em gado de corte. In: González, F.H.D.; Ospina, H.; Barcelos, J.O. e Ribeiro, L.A.O. (Eds.). Perfil metabólico em ruminantes: Seu uso em nutrição e doenças nutricionais. Gráfica UFRGS. Porto Alegre.

González F.H.D. e Silva S.C. 2006. Introdução a bioquímica clínica veterinária, $2^{\circ}$ edição. Editora da UFRGS. Porto Alegre.

Greenwood, P.L.; Hunt, A.S.; Hermanson, J.W. and Bell, A.W. 2000. Effects of birth weight and post natal nutrition on neonatal sheep. II. Skeletal muscle growth and development. J Anim Sci, 78: 50-61.

Hauner, H. 1995. Nutrition and metabolic syndrome. Internist (Berl), 36: 1040-1045.

Husted, S.M.; Nielsen, M.O.; Blache, D. and Inggavarrtsen, K.L. 2008. Glucose homeostasis and metabolic adaptation in the pregnant and lactating sheep are affected by the level of nutrition previously provided during her late fetal life. Domest Anim Endocrinol, 34: 419-431.

Jawerbaum, A. and White, V. 2010. Animal models in diabetes and pregnancy. Endocr Rev, 31: 680-701.

Lostroh, A.J. and Krahl. M.E. 1973. Insulin action. Accumulation in vitro of $\mathrm{Mg} 2+$ and $\mathrm{K}+$ in rat uterus: ion pump activity. Biochim Biophys Acta, 291: 260-268.

Lee, C.T.; Lien, Y.H.H.; Lei. L.W.; Chen, J.B.; Lin, C.R. and Chen, H.C. 2006. Increased renal calcium and magnesium transporter abundance in streptozotocin-induced diabetes mellitus. Kidney Int, 69: 1786-1791.

Mcnair, P.; Christiansen, C.; Madsbad, S.; Lauritzen, E.; Faber, O.; Binder, C. and Transbol, I. 1978. Hypomagnesemia, a risk factor in diabetic retinopathy. Diabetes, 27: 1075-1077.

Mexia, A.A.; Macedo, F.A.F.; Macedo, R.M.G.; Sakaguti, E.S.; Santello, G.A.; Capovilla, L.C.T.; Zund, M. e Sasa, A. 2006. Desempenho e características das fibras musculares esqueléticas de cordeiros nascidos de ovelhas que receberam suplementação alimentar em diferentes períodos da gestação. Rev Bras Zootecn, 35: 1780-1787.

Mooren, F.C.; Kruger, K.; Volker, K.; Golf, S.W.; Wadepuhl, M. and Kraus. A. 2011. Oral magnesium supplementation reduces insulin resistance in non-diabetic subjects - a double-blind, placebo-controlled, randomized trial. Diabetes Obes Metab, 13: 281-284.

Montossi, F.; Juliän, R.S. y Mattos, D. 1998. Alimentación y manejo de la oveja de cria durante el último tercio de gestación en la región de basalto. In: seminario de actualizacion em tecnologias para basalto, Tacuarembó. Anais... INIA. Tacuarembó. (Serie Técnica, 102).

Mulligan, F.J.; O'grady, L.; Rice. D.A. and Doherty. M.L. 2006. A herd health approach to dairy cow nutrition and production diseases of the transition cow. Anim Reprod Sci, 96: 331-353.

Nasciutti, N.R. 2011. Perfil metabólico em ovelhas Santa Inês com baixo escore de condição corporal no periparto. 2011. Dissertação (Mestrado). Universidade Federal de Uberlândia. Uberlândia. $41 \mathrm{f}$.

Nikolajczyk, B.S.; Jagannathan-Bogdan, M.; Shin, H. and Gyurko, R. 2011. State of the union between metabolism and the immune system in type 2 diabetes. Genes Immun, 12: 239-50.

Nyholm, B.; Qu, Z.; Kaal, A.; Pedersen, S.B.; Gravholt, C.H.; Andersen, J.L.; Saltin, B. and Schmitz, O. 1997. Evidence of an increased number of type $\mathrm{llb}$ muscle fibers in insulin-resistant first-degree relatives of patients with NIDDM. Diabetes, 46: 1822-1828.

Oberbach, A.; Bossenz, Y.; Lehmann, S.; Niebaver, J.; Adams, V.; Paschke, R.; Schon, M.R.; Bluher, M. and Punkt, K. 2006. Altered fiber distribution and fiber-specific glycolytic and oxidative enzyme activity in skeletal muscle of patients with type 2 diabetes. Diabetes Care, 29: 895-900.

Oikawa, S. and Oetzel, G.R. 2006. Decreased insulin response in dairy cows following a four-day fast to induce epatic lipidosis. J Dairy Sci, 89: 2999-3005. 
Patiño, P.R. e Van Cleef, E. 2010. Aspectos fundamentales del crecimiento em ovinos. Rev Colombiana Cienc Anim, 2: 399-421.

Payne, J.M. and Payne, S. 1987. The metabolic profile test. Oxford University Press. Oxford.

Peixoto, L.A. e Osório, M.T. 2007. Perfil metabólico protéico e energético na avaliação do desempenho reprodutivo em ruminantes. Rev Bras Agrociência, 13: 299-304.

Pugh, D.G. 2005. Clínica de ovinos e caprinos. Roca. São Paulo. 511 pp. Rabassa, V.R.; Tabeleão, V.C.; Schneider, A.; Menezes L.M.; Severo, S.; Schwegler, E.; Goulart, M.A.; Del Pino, F.A.B.; Nogueira, C.E.W. e Corrêa, M.N. 2009. Avaliação metabólica de ovelhas de cria mantidas em campo nativo durante o período de outono/inverno. Rev Bras Agrociência, 15: 125-128.

Ramin, A.G.; Asri-Rezaie, S. and Macali, S.A. 2007. An evaluation of serum glucose, bhb, urea and cortisol concentrations in pregnant ewes. Folia Vet, 51: 9-13.

Regnault,T.R.; Oddy, H.V.; Nancarrow, C.; Sriskandarajah, N. and Scaramuzzi, R.J. 2004. Glucose-stimulated insulin response in pregnant sheep following acute suppression of plasma non-esterified fatty acid concentrations. Reprod Biol Endocrinol, 2: 64.

Ribeiro, L.A.O.; González, F.H.D.; Conceição, T.R.; Brito, A.B.; La Rosa, V.L. e Campos, R. 2003. Perfil metabólico de borregas Corriedale em pastagem nativa do Rio Grande do Sul. Acta Scie Vet, 31: 167-170.

Ribeiro, L.A.O.; Mattos, R.C.; González, F.H.D.; Wald, V.B.; Silva, M.A. e La Rosa, V.L. 2004. Perfil metabólico de ovelhas Border Leicester $x$ Texel durante a gestação e a lactação. Rev PortCienc Vet, 99: 155-159.
Roche, J.R.; Friggens, N.C; Kay, J.K.; Fisher, M.W.; Stafford, K.J. and Berry, D.P. 2009. Invited review: Body condition score and its association with dairy cow productivity, health, and welfare. J Dairy Sci, 92: 5769-5801.

Russel, A.J.F. 1979. The nutrition of the pregnant ewe. In: The management and diseases of sheep. British Council. Edinburg.

Santos, F.C.O.; Mendonça, C.L.; Silva Filho, A.P.; Carvalho, C.C.D.; Soares, P.C. e Afonso, J.A.B. 2011 . Indicadores bioquímicos e hormonais de casos naturais de toxemia da prenhez em ovelhas. Pesquisa Vet Brasil, 31: 974-980.

Schulumbohm, C. and Harmeyer, J. 2003. Hypocalcemia reduces endogenous glucose production in hyperketonemic sheep. J Dairy Sci, 86: 1953-1962.

Smith, M.C. and Sherman, D. 2009. Goat medicine. $2^{\text {nd }}$ ed. Lea and Febiger. Philadelphia. $871 \mathrm{pp}$

Venoiärvi, M.; Puhke, R.; Hämäläinen, H.; Marniemi, J.; Rastas M.; Rusko H.; Nuutila, P.; Hänninen, O. and Aunola, S.; 2005. Role of skeletal muscle-fibre type in regulation of glucose metabolism in middle-aged subjects with impaired glucose tolerance during a long-term exercise and dietary intervention. Diabetes Obes Metab, 7: 745-754.

Venu, L.; Padmavathi, I.J.N.; Kishore, Y.D.; Bhanu, N.V.; Rao, K.R.; Sainath, P.B.; Ganeshan, M. and Raghunath, M. 2008. Long-term effects of maternal magnesium restriction on adiposity and insulin resistance in rat pups. Obesity, 16: 1270-1276. 